\title{
A checklist for zooplankton (Rotifera, Copepoda, Cladocera) of European Turkey inland waters
}

\section{Türkiye Avrupası'nın iç sularında yaşayan zooplankton (Rotifera, Copepoda, Cladocera) türlerinin listesi}

\section{Hüseyin Güher}

Trakya University, Faculty of Science, Department of Biology, Edirne, TR-22030, Turkey

huseying@trakya.edu.tr

How to cite this paper:

Güher, H., 2014 A Checklist for zooplankton (Rotifera, Copepoda, Cladocera) of European Turkey inland waters. Ege J Fish Aqua Sci 31(4): 221-225.

doi: 10.12714/egejfas.2014.31.4.08

Özet: Türkiye'nin Avrupa kısmı, Avrupa ile Asya kıtalar arasında geçiş bölgesi oluşturması nedeniyle her iki kıtanında türlerine ev sahipliği yapmaktadır. Ancak şu ana kadar Anadoludan ayrı olarak Türkiye Avrupası zooplanktonik organizmaların tür listesi çıkarılmamıştır. Bu nedenle bölgede bu güne kadar bu konuda yapılan araştırmaların tümü incelenmiştir. 1940'tan bu yana yapılan araştırmaların incelenmesi sonucunda 138 Rotifera, 53 Copepoda ve 65 Cladocera olmak üzere 256 tür listelenmiştir.

Anahtar kelimeler: Tür listesi, Zooplankton, içsular, Türkiye Avrupası

Abstract: The European part of Turkey, which constitutes a transitional region between Europe and Asia hosts species of both continents. But, the zooplankton checklist in the European part of Turkey has not been published so far as a separate checklist from that of Turkey as a whole. Therefore, a checklist was presented in this study based on compilation of previous zooplankton studies carried out at European Turkey inland waters. As a result of the studies conducted since 1940 to date, a total of 256 taxa belonging to 138 Rotifera, 53 Copepoda and 65 Cladocera were listed.

Keywords: Checklist, Zooplankton, inland waters, EuropeanTurkey

\section{INTRODUCTION}

Zooplanktonic organisms are not only the main food source of fishes that live in fresh water ecosystems, but also they include indicator species which is used as an indication of water quality, pollution and the state of eutrophication. For his reason, many researches have been conducted in terms of both taxonomical and ecological aspects of zooplanktonic organisms.

Studies performed in order to determine the zooplankton fauna of Turkey continued over the years without stopping. Some of these studies focused on Rotifera and Cladocera or Copepoda. The first checklist on Cladocera was published by Gündüz (1997) and the second one on Rotifera was published by Ustaoglu et al. (2012a). In addition, the checklist for zooplankton of Turkish inland waters listed 229 Rotifera, 92 Cladocera and 106 Copepoda species previos in studies (Ustaoğlu, 2004) and this list was updated by Ustaoğlu (2014). Moreover, checklist for zooplankton of Eastern and Souteastern Anatolia Regions (Turkey) was published by Bulut and Saler (2014). But, the zooplankton checklist in the
European part of Turkey has not been published so far as a separate checklist from that of Turkey as a whole.

Inventory works on zooplankton diversity of European Turkey inland waters were carried out within the past 75 years and the first research began in the 1940. However, these researches progressed slowly and gained acceleration after 1990.

This study aims to present a current checklist of zooplankton (Rotifera, Copepoda, Cladocera) in European Turkey inland waters, based on compilation of previous inventory studies carried out so far.

\section{MATERIALS AND METHODS}

European part of Turkey covers an area of $23.764 \mathrm{~km}^{2}$ and creates a crossing point between Europe and Asia continents. Kırklareli, Edirne and Tekirdag province are located in the region and Çanakkale and Istanbul are partly represented (Figure1). 


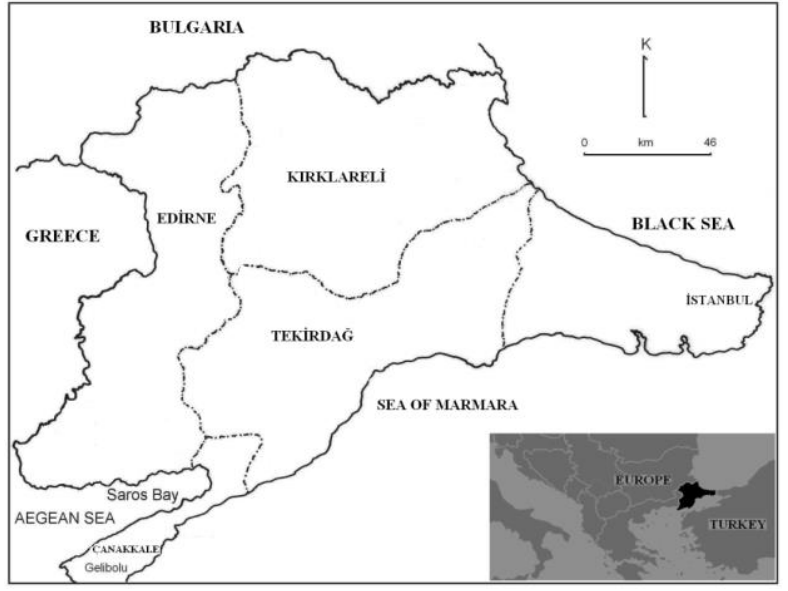

Figure 1. Map of European Turkey.

In this study, all published literature, either papers or theses from 1940 to 2012 were examined. Taxonomical species and author names were written according to Segers (2007), Ustaoğlu (2004), Ustaoğlu et al. (2012a) and Gündüz (1997). The studies performed concerning the zooplankton of European Turkey inland waters are as follows: Mann (1940), Muckle (1951), Kiefer (1952, 1955), Lindeberg (1953), Demirhindi (1972), Fiers (1979), Ortak and Kırgız (1988), Güher and Kırgız (1989, 1992, 1994, 2004, 2007, 2008), Gündüz (1997), Güher (1999, 2000, 2002, 2003, 2004, 2012), Güher et al. (2004, 2011), Erdoğan and Güher (2005, 2008, 2012a, 2012b), Okgerman et al. (2007), Güher and Erdoğan (2008), Özçalkap and Temel (2011), Dorak and Albay (2011), Ustaoğlu et al. (2012b)

\section{RESULTS AND DISCUSSION}

As a result of the throughout investigation of the studies in European Turkey inland waters from 1940 to 2012, the presence of a total 256 taxa was reported. Among these, Rotifera was represented with 138 species belonging to 25 families, Copepoda with 53 species belonging to 11 families and Cladocera with 65 species belonging to 10 families. When the recorded taxa were grouped according to their presence in terms of province borders in the region, it appeared that 208 species existed in Edirne, 158 species in Kırklareli, 118 species in Tekirdağ and 107 species in Istanbul. Çanakkale is missing in this distribution because no study was performed in Gallipoli Peninsula (Çanakkale), the part of the province in European of Turkey. These species and their distributions have been shown at below (E: Edirne; $\mathrm{K}$ : Kırklareli; $\mathrm{T}$ : Tekirdağ: I: İstanbul).

\section{Phylum: ROTIFERA}

Classis: Eurotatoria De Ridder, 1957

Subclassis: Bdelloidea Hudson, 1884

Family: Philodinidae Ehrenberg, 1838 Dissotrocha aculeata (Ehrenberg, 1832): E,T Philodina megalotrocha Ehrenberg, 1832: E, K, T Rotaria neptunia (Ehrenberg, 1830): E, K, T Rotaria rotatoria (Pallas, 1766): E

Family: Adinetidae Hudson \& Gosse, 1889 Adineta sp.: K
Family: Habrotrochidae Bryce, 1910 Habrotrocha sp.: E, K, T

Subclassis: Monogononta Plate, 1889

Superorder: Pseudotrocha Kutikova, 1970

Order: Ploimia Hudson \& Gosse, 1886

Family: Epiphanidae Harring, 1913 Epiphanes macroura (Barrois \& Daday, 1894): E,I Epiphanes senta (Müller, 1773): E

Proalides subtilis Rodewald, 1940: E Proalides tentaculatus De Beauchamp, 1907: E

Family: Brachionidae Ehrenberg, 1838 Anuraeopsis coelata De Beuchamp, 1932: K, I Anuraeopsis fissa Gosse, 1851: E, K, T, I Anuraeopsis navicula Rousselet, 1911: E, K Brachionus angularis Gosse, 1851: E, K, T, Brachionus bidentatus Anderson, 1889: E, Brachionus budapestinensis Daday, 1885: E, K, I Brachionus calyciflorus Pallas, 1766: E, K, T, I Brachionus diversicornis (Daday, 1883): E, K, T, I Brachionus falcatus Zacharias, 1898: E, K, T, I Brachionus forficula Wierzejski, 1891: E Brachionus leydigii Cohn, 1862: E, K, T Brachionus plicatilis Müller, 1786: E, K, I Brachionus quadridentatus Hermann, 1783: E, K, T, I Brachionus urceolaris Müller, 1773: E, K, T, I Kellicottia longispina (Kellicott, 1879): E, K Keratella cochlearis (Gosse, 1851): E, K, T, I Keratella quadrata (Müller, 1786): E, K, T, I

Keratella tecta (Gosse, 1851): E, K, T Keratella tropica (Apstein, 1907): E, K, T Notholca acuminata (Ehrenberg, 1832): E, K, T, Notholca salina Focke, 1961: E Notholca squamula (Müller, 1786): E, K, T, Plationus patulus (O.F.Müller, 1786): E, K Platyias quadricornis (Ehrenberg, 1832): E, K

Family: Euchlanidae Ehrenberg, 1838

Euchlanis deflexa (Gosse, 1851): E, K, T Euchlanis dilatata Ehrenberg, 1832: K, T, I Euchlanis incisa Carlin, 1939: E, K, T Euchlanis lyra Hudson, 1886: E, K, T Euchlanis meneta Myers, 1930: E, K

Family: Mytilinidae Harring, 1913 Lophocharis salpina (Ehrenberg, 1834): E, K, T Mytilina mucronata (Müller, 1773): E, K Mytilina ventralis (Ehrenberg, 1830): $\mathrm{E}$

Family: Trichotriidae Harring, 1913 Trichotria pocillum (Müller, 1776): E, K Trichotria tetractis (Ehrenberg, 1830): E, K, T

Family: Lepadellidae Harring, 1913 Colurella adriatica Ehrenberg, 1831: E, K, T, I Colurella colurus (Ehrenberg, 1830): E, K, T, I Colurella obtusa (Gosse, 1886): E Colurella uncinata (Müller, 1773): E, K, T Lepadella (Heterolepadella) ehrenbergi (Perty, 1850): T Lepadella (Lepadella) acuminata (Ehrenberg, 1834) : E, K Lepadella (Lepadella) patella (Müller, 1773): E, K, T, Lepadella (Lepadella) triptera (Ehrenberg, 1832): E Lepadella(Lepadella) ovalis (Müller, 1786): E, K Squatinella rostrum (Schmarda, 1846): E

Family: Lecanidae Remane, 1933 Lecane bulla (Gosse, 1886): E, K, T

Lecane closterocerca (Schmarda, 1859): E, K, T Lecane donneri Chengalath \& Mulamoottil, 1974: E Lecane flexilis (Gosse, 1886): $\mathrm{E}$ Lecane furcata (Murray, 1913): E, K, T Lecane hamata (Stokes, 1896): E, K, T Lecane hastata (Murray, 1913): E, T Lecane inermis (Bryce, 1892): E Lecane lamellata (Daday, 1893): E, K Lecane luna (Müller, 1776): E, K, T, I Lecane lunaris (Ehrenberg, 1832): E, K, T 
Lecane nana (Murray, 1913): E, K, I

Lecane pyriformis (Daday, 1905): E, K, T Lecane quadridentata (Ehrenberg, 1830): E, T

Lecane rugosa (Harring, 1914): E

Lecane stenroosi (Meissner, 1908): E, K, T

Lecane stichaea Harring, 1913: K

Lecane ungulata (Gosse, 1887): E, K, T

Family: Proalidae Harring \& Myers, 1924

Proales fallaciosa Wulfert, 1937: E, K, T

Family: Notommatidae Hudson \& Gosse, 1886

Cephalodella catellina (Müller, 1786): E,

Cephalodella forficula (Ehrenberg, 1830): E, K

Cephalodella gibba (Ehrenberg, 1830): E, K, T, I

Cephalodella megalocephala (Glascott, 1893): E, T

Eosphora ehrenbergi Weber \& Montet, 1918: K

Monommata sp.: E, K

Notommata copeus Ehrenberg, 1834: E

Notommata glyphura Wulfert, 1935: E, K, T

Pleurotrocha petromyzon (Ehrenberg, 1830): E, K, T

Family: Ituridae Sudzuki, 1964

Itura myersi Wulfert, 1935: E, K, T

Family: Scaridiidae Manfredi, 1927 Scaridium longicaudum (Müller, 1786): E

Family: Trichocercidae Harring, 1913

Trichocerca bicristata (Gosse, 1887): E, K

Trichocerca capucina (Wierjeski \& Zacharias, 1893): E, K, T

Trichocerca cylindrica (Imhof, 1891): E, K, T, I

Trichocerca elongata (Gosse, 1886): E

Trichocerca iernis (Gosse, 1887): K

Trichocerca insignis (Herrick, 1885): $\mathrm{K}$

Trichocerca longiseta (Schrank, 1802): K

Trichocerca obtusidens (Olofsson, 1918): E

Trichocerca porcellus (Gosse, 1851): E, K, T, I

Trichocerca pusilla (Jennings, 1903): E, K, T

Trichocerca rattus (Müller, 1776): E, K

Trichocerca similis (Wierzejski, 1893): E, K

Trichocerca tenuior (Gosse, 1886): E, K

Trichocerca tigris (Müller, 1786): E, K

Family: Gastropodidae Harring, 1913

Ascomorpha ecuadis Petry, 1850: I

Ascomorpha ovalis (Bengendahl, 1892): E, I

Ascomorpha saltans Bartsch, 1870: E

Gastropus minor (Rousselet, 1892): E

Gastropus stylifer (Imhof, 1891): I

Family: Synchaetidae Hudson and Gosse, 1886

Polyarthra dolichoptera Idelson, 1925: E, K, T, I

Polyarthra euryptera Wierzejski, 1891: E

Polyarthra major Burckhardt, 1900: I

Polyarthra remata Skorikov, 1896: E, K, T

Polyarthra vulgaris Carlin, 1943: E, K, T, I

Synchaeta oblonga Ehrenberg, 1832: E, K, T,

Synchaeta pectinata Ehrenberg, 1832: E, K, T,

Synchaeta stylata Wierzejski, 1893: K, I

Family: Asplanchnidae Eckstein, 1883

Asplanchna girodi de Guerne, 1888: E

Asplanchna priodonta Gosse, 1850: E, K, T, I

Asplanchna sieboldi (Leydig, 1854): E, K, T, I

Asplanchnopus hyalinus Harring, 1913: E

Family: Dicranophoridae Harring, 1913

Dicranophoroides caudatus (Ehrenberg, 1834): $\mathrm{K}$ Dicranophorus grandis (Ehrenberg, 1832): E, K, T

Encentrum kulmatyckii Wiszniewski, 1953: E

Encentrum saundersiae (Hudson, 1885): E, K

Erignatha clastopis (Gosse, 1886): K

Paradicranophorus hudsoni (Glascott, 1893): E, K, T

Superordo: Gnesiotrocha Kutikova, 1970

Order: Flosculariacea Harring, 1913

Family: Flosculariidae Ehrenberg, 1838 Floscularia sp.: E, K, T

Family: Testudinellidae Harring, 1913 Pompholyx sulcata Hudson, 1885: E, K, T,
Testudinella elliptica (Ehrenberg, 1934): E

Testudinella emarginula (Stenroos, 1898): E, K

Testudinella mucronata (Gosse, 1886): E

Testudinella parva (Ternetz, 1892): T

Testudinella patina (Hermann, 1783): E, K, T, I

Family: Filiniidae Harring \& Myers, 1926

Filinia cornuta (Weisse, 1847): E

Filinia longiseta (Ehrenberg, 1834): E, K, T,

Filinia opoliensis (Zacharias, 1898): E, K, T

Filinia terminalis (Plate, 1886): E, K, T, I

Family: Conochilidae Harring, 1913

Conochilus (Conochiloides) dossuarius Hudson, 1885: E, K, T

Conochilus (Conochilus) unicornis Rousselet, 1892: K, T

Family: Hexarthridae Bartos, 1959

Hexarthra fennica (Levander, 1892): E, T

Hexarthra intermedia (Wiszniewski, 1929): I

Hexarthra mira (Hudson, 1871): E, K, T, I

Order: Collothecaceae Harring, 1913

Family: Collothecidae Harring, 1913

Collotheca ornata (Ehrenberg, 1832): E, K, T

\section{Subclassis: COPEPODA}

Order: Cyclopoida Sars, 1918

Family: Cyclopidae G.O. Sars, 1913

Subfamily: Eucyclopinae Kiefer, 1927

Ectocyclops phaleratus (Koch, 1838): E, K

Eucyclops macruroides (Lilljeborg, 1901): E, K

Eucyclops macrurus (G.O.Sars, 1863): E, T,

Eucyclops serrulatus (Fischer, 1851): E, K, T,

Eucyclops speratus (Lilljeborg, 1901): E, K, T

Macrocyclops albidus (Jurine, 1820): E, K, T,

Macrocyclops fuscus (Jurine, 1820): E, K, T

Paracyclops affinis (G.O.Sars, 1863): E, K, T

Paracyclops fimbriatus (Fischer, 1853): T, I

Tropocyclops prasinus (Fischer, 1860): T, I

Subfamily: Cyclopinae Kiefer, 1927

Acanthocyclops kieferi (Chappuis, 1925): E

Acanthocyclops robustus (G.O.Sars, 1863): E, K, T, I

Acanthocyclops venustus (Norman \& Scoott, 1906): E, K, T

Cyclops abyssorum G.O.Sars, 1863: E, K, T,

Cyclops furcifer Claus, 1857: E

Cyclops insignis Claus, 1857: E

Cyclops kolensis Lilljeborg, 1901: K

Cyclops scutifer G.O.Sars, 1863: K, T, I

Cyclops strenuus Fischer, 1851: E, K, T,

Cyclops vicinus Uljanin, 1875: E, K, T, I

Diacyclops bicupidatus odessanus (Schmankevitch, 1875): I

Diacyclops bicuspidatus (Claus, 1857): E, K

Diacyclops bisetosus (Rehberg, 1880): I

Megacyclops gigas (Claus, 1857): E, K, T

Megacyclops latipes (Lowndes, 1927):

Megacyclops viridis (Jurine, 1820): E, K, T, I

Mesocyclops leuckarti (Claus, 1857): K,

Metacyclops gracilis (Lilljeborg 1853): E,

Metacyclops planus (Gurney, 1909): E

Thermocyclops crassus (Fischer, 1853): E, K,

Thermocyclops dybowskii (Landé, 1890): E

Subfamily: Halicyclopinae Kiefer, 1927

Halicyclops neglectus Kiefer, 1935: E, K

Family: Oithonidae, Dana, 1853

Oithona nana Giesbrecht, 1893: E

Order: Calanoida Sars, 1930

Family: Acartiidae G.O.Sars, 1903

Acartia (Acartiura) clausi Giesbrecht, 1889: E

Family: Pseudodiaptomidae G.O.Sars, 1903

Calanipeda aquedulcis Kritschagin, 1873: E, K, T, I

Family: Diaptomidae G.O.Sars, 1903

Subfamily: Diaptominae Kiefer, 1932

Arctodiaptomus belgrati (Mann, 1940): I

Arctodiaptomus byzantinus Mann, 1940: I 
Arctodiaptomus pectinicornis (Wierzejski, 1887): I

Arctodiaptomus wierzejskii (Richard, 1888): E, K, T

Hemidiaptomus brehmi (Mann, 1940): I

Mixodiaptomus kupelwieseri (Brehm, 1907): K, I

Family: Temoridae G.O.Sars, 1903

Eurytemora velox (Lilljeborg, 1853): K, I

Order: Harpacticoida Sars, 1911

Family: Harpacticidae Dana, 1846

Harpacticus sp.: E

Family: Canthocamptidae Sars, 1906

Attheyella trispinosa (Brady, 1880): I

Attheyella wulmeri De Kerherve, 1914: I

Bryocamptus pygmaeus (G.O.Sars, 1863): I

Canthocamptus microstaphylinus Wolf, 1905: E, K, I

Family: Laophontidae T.Scott, 1904

Heterolaophonte stroemii (Baird, 1834): E

Onychocamptus mohammed (Blanchard, Rıchard, 1891): E, K, I

Family: Ameiridae (Monard, 1936)

Nitocra hibernica (Brady, 1880): E, K, T, I

Nitocra lacustris (Schmanevitsch, 1875): E

Family: Dacythompsoniidae Lang, 1936

Leptocaris brevicornis (Van Douwe, 1904): E

Leptocaris trisetosa (Kunz, 1935): E

\section{Suborder: CLADOCERA}

Family: Daphniidae Sars, 1865

Ceriodaphnia dubia Richard, 1894: E

Ceriodaphnia laticaudata P.E.Müller, 1867: E

Ceriodaphnia megops Sars, 1862: K

Ceriodaphnia quadrangula (O.F.Müller, 1758): E, K, T, I

Ceriodaphnia reticulata (Jurine, 1820): E, K, T, I

Daphnia (C.) atkinsoni Baird, 1859: E, I

Daphnia (C.) magna Straus, 1820: E

Daphnia (C.) similis Claus, 1876: E, K, T

Daphnia (C.) ulomskyi Benning 1914: E

Daphnia carinata King, 1852: I

Daphnia cucullata Sars, 1862: E

Daphnia curvirostris Eylmann, 1887: I

Daphnia galeata Sars, 1864: E

Daphnia hyalina Leydig, 1860: E,I

Daphnia longispina O.F.Müller, 1785: E, K, T, I

Daphnia obtusa Kurz, 1874: E, K, T, I

Daphnia parvula Fordyce, 1901: E

Daphnia pulex Leydig, 1860: E, K, T, I

Megafenestra aurita (Fischer,1849): E, K

Scapholeberis kingi Sars, 1903: E, K, T, I

Scapholeberis mucronata (O.F.Müller,1758): E, K, I

Simocephalus exspinosus (Koch, 1841): E, K, T

Simocephalus serrulatus (Koch, 1841): E, T,

Simocephalus vetulus (O.F.Müller, 1776): E, K, T, I

In studies conducted in the Palaearctic Region, 1348 Rotifera, 245 Cladocera and 1204 Copepoda species were identified so far (Segers, 2008; Forro et al. 2008; Boxshall and Defaye, 2008) whereas these groups in Turkey are represented with 378,98 and 135 species, respectively
Family: Sididae (Baird, 1850)

Diaphanosoma birgei Korinek, 1981: E, T

Diaphanosoma brachyurum, (Liévin, 1848): E, K, T,

Diaphanosoma orghidani Negrea, 1982: E, T

Family: Bosminidae (Baird, 1845)

Bosmina coregoni Baird, 1857: E

Bosmina longirostris (O.F.Müller, 1785): E, K, T, I

Family: Chydoridae Stebbing, 1902

Acroperus harpae (Baird, 1835): K, I

Alona costata Sars, 1862: E, K, I

Alona guttata Sars, 1862: E, K, I

Alona protzi Hartwig, 1900: I

Alona rectangula Sars, 1862: E, K, T, I

Alonella excisa (Fischer, 1854): E, K, T

Biapertura affinis (Leydig, 1860): E, K,

Biapertura intermedia (Sars, 1862): E

Camptocercus rectirostris Schoedler, 1862: K

Chydorus latus Sars, 1862: E, I

Chydorus sphaericus (O.F.Müller; 1776): E, K, T, I

Coronatella quadrangularis (O.F.Müller, 1785): E, K, T, I

Disparalona rostrata (Koch, 1841): E, T

Dunhevedia crassa King, 1853: E

Graptoleberis testudinaria (Fischer, 1848): K, I

Leydigia acanthocercoides (Fischer, 1854): E, I

Leydigia leydigi (Schoedler, 1863): E, K, T

Oxyurella tenuicaudis (Sars, 1862): $\mathrm{E}$

Picripleuroxus laevis (Sars, 1862): K

Pleuroxus aduncus (Jurine, 1820): E, K, T, I

Pleuroxus truncatus (O.F.Müller, 1785): E, K

Tertocephala ambigua (Lilljeborg, 1900): E

Family: Moinidae Goulden, 1968

Moina brachiata (Jurine, 1820): E, K, T

Moina macrocopa (Straus, 1820): T

Moina micrura Kurz, 1874: E, K, T

Moina salina Daday, 1888: E, T

Family: Macrothricidae Norman \& Brady, 1867

llyocryptus sordidus (Liévin, 1848): E, K, T, I

Lathonura rectirostris (O.F.Müller, 1785): I

Ilyocrptus agilis Kurz, 1878: E, K, T, I

Macrothrix hirsuticornis Norman \& Brady, 1867: E, K

Macrothrix laticornis (Fischer, 1851): E, K, T, I

Macrothrix rosea Lievin, 1848: E

Family: Leptodoridae Lilljeborg, 1861

Leptodora kindtii (Focke, 1844): E, I

Family: Polyphemidae Barid, 1845

Polyphemus pediculus (Linnaéus, 1761): K

Family: Cercopagidae Mordukhai-Boltovskoi, 1968 Cercopagis pengoi (Ostroumov, 1892): I

Family: Podonidae Mordukhai-Boltovskoi, 1968 Cornigerius meaticus (Pengo, 1879): I

(Gündüz, 1997; Ustaoğlu 2004, 2014, Ustaoğlu et al. 2012a) (Table 1). The European part of Turkey, which constitutes a transitional region between Europe and Asia, hosts species of both sides which in turn leads to an increased diversity in this part of Turkey.

Table 1. Number of Rotifera, Cladocera and Copepoda species currently known in Palaearctic and Turkey.

\begin{tabular}{llllll}
\hline & & PALAEARCTIC & \multicolumn{3}{c}{ TURKEY } \\
\hline & $\begin{array}{l}\text { Number } \\
\text { species }\end{array}$ & \multicolumn{1}{c}{ Reference } & $\begin{array}{l}\text { Number } \\
\text { species }\end{array}$ & Reference & $\begin{array}{l}\text { EUROPEAN } \\
\text { TURKEY }\end{array}$ \\
Rotifera & 1348 & Segers (2008) & 378 & $\begin{array}{l}\text { Ustaoğlu et al. (2012a) } \\
\text { Ustaoğlu (2014) }\end{array}$ & 138 \\
Cladocera & 245 & Forro et al. (2008) & 98 & $\begin{array}{l}\text { Gündüz }(1997) \\
\text { Ustaoğlu }(2004,2014)\end{array}$ & 65 \\
Copepoda & 1204 & Boxshall and Defaye (2008) & 135 & Ustaoğlu $(2004,2014)$ & 53 \\
Total & 2797 & & 611 & & 256 \\
\hline
\end{tabular}




\section{REFERENCES}

Boxshall, G.A., Defaye, D., 2008. Global diversity of copepods (Crustacea: Copepoda) in freshwater. Hydrobiologia 595:195-207. doi: 10. 1007/s10750-007-9014-4

Bulut, H., Saler, S., 2014. A Checklist for zooplankton of Eastern and Souteastern Anatolia regions (Turkey). Düzce University Journal of Science \& Technology 2: 36-47.

Demirhindi, Ü., 1972. The Preliminary planktonic investigations in the coasta lagoons and several brackish water lakes of Turkey. l.Ü.Fen. Fak. Mec. 37 (3-4): 205-232.

Dorak, Z., Albay, M., 2011. Streams of Alibeyköy and Eyüp (Istanbul) determination of the some limnological characteristics and investigation of effect on estuary ecosystem (in Turkish). X. Ulusal Ekoloji ve Çevre Kongresi 04-07 Ekim 2011, Çanakkale, p.64.

Erdoğan, S., Güher, H. 2005., The Rotifera fauna of Gala lake (EdirneTurkey). Pakistan. Journal of Biological Sciences, 8 (11),1579-1583.

Erdoğan, S., Güher, H.,2008. An investigation on the periphytic species of Rotifera in the lake Gala (Turkey). Acta Zoologica Bulgarica, 60 (1): 31 39.

Erdoğan, S., Güher, H., 2012a. The Rotifera fauna of Turkish Thrace (Edirne Tekirdağ, Kırklareli). Journal of Fisheries Sciences.com. 6(2):132-149. doi: 10.3153/jfscom.2012017

Erdoğan, S., Güher, H., 2012b. Four new Rotifera species of Turkish fauna Turkish Journal of Fisheries and Aquatic Sciences,12:165-169. doi: 10.4194/1303-2712-v12_1_19

Fiers, F., 1979. Bijdrage tot de limnologische kennis van Turkije met nadruk op de Entomostraca (Crustacea). Rijksuniversiteit Ghent, Faculteit der Wetenschappen Afdeling Biologie Groep Dierkunde, $119 \mathrm{p}$.

Forro, L., Korovchinsky, N.M. Kotov, A.A., Petrusek, A., 2008. Global diversity of cladocerans (Cladocera; Crustacea) in freshwater. Hydrobiologia, 595:177-184 doi: 10.1007/s10750-007-9013-5

Güher, H., 1999. A taxonomical study on the Cladocera and Copepoda species (Crustacea) of Mert, Erikli, Hamam, Pedina lakes (Iğneada/Kırklareli) (in Turkish with English abstract). Tr. J. of Zoology, 23, Eksayı 1, 47-53.

Güher, H. 2000. A faunistic study on the freshwater Cladocera (Crustacea) species in Turkish Thrace (Edirne, Tekirdağ, Kırklareli). Tr. J. Zoology, 24, 237-243.

Güher, H., 2002. Cladocera and Copepoda (Crustacea) Fauna of Lake Terkos (Durusu). Turk J. Zool. 26, 283-288

Güher, H., 2003. Community structure of zooplanktonic organisims in Mert, Erikli, Hamam, and Pedina (Iğneada/Kırklareli) Lakes (in Turkish with English abstract). Ege J Fish Aqua Sci 20 (1-2): 51-62

Güher, H., 2004. A study on morphological characters, spatial and seasonal densities, and co-existence of two predatory Cladocera, Cercopagis pengoi (Ostroumov, 1891) and Cornigerius meaticus (Pengo, 1879) in Lake Terkos, Turkey. Crustaceana, Volume 77, No. 6, 669-681.

Güher, H., 2012. The investigation of zooplanktonic organisms (Rotifera Copepoda, Cladocera) of Meriç River (Turkey). Journal of Animal and Veterinary Advances, 11 (24): 4673-4677

Güher, H., Erdoğan S., 2008. An investigation on the periphytic zooplankton species (Cladocera, Copepoda, Rotifera) in Alıc pond (Turkey) (in Turkish with English abstract). Journal of Fisheries Sciences.com. 2(3): 516-523. doi: 10.3153/jfscom.mug.200749

Güher, H., Erdoğan, S., Kırgız, T., Camur-Elipek, B. 2011. Dynamics of zooplankton in National Park of Lake Gala (Edirne-Turkey). Acta Zoological Bulgarica 63 (2), 157-168.

Güher, H., Kırgız, T., 1989. Cladocera and Copepoda species of Süleoğlu dam lake and Korucuköy, Budakdoğanca, Eskikadın ponds (in Turkish with English abstract). Anadolu Üniv. Fen Ed Fak. Der. C.2, S. 1, 25-43.
Güher, H., Kırgız, T., 1992. Edirne province Cladocera (Crustacea) species (in Turkish). Firat Univ., XI. Ulusal Biyoloji Kongresi, Hidrobiyoloji Sek., 24-27 Haziran 1992, Elazığ, 89-97.

Güher, H., Kırgız, T., 1994. Edirne province freshwater Copepoda (Crustacea) species and their distributions (in Turkish). Trakya Univ., XII. Ulusal Biyoloji Kongresi, Hidrobiyoloji Sek, 6-8 Temmuz 1994, Edirne, 220-226.

Güher, H., Kırgız, T., 2004. The Copepoda (Crustacea) freshwater fauna of Turkish Thrace region (Edirne, Kırklareli, Tekirdağ). Pakistan Journal of Biological Sciences, 7 (5), 834-837.

Güher, H., Kırgız, T., 2007. A study on associated microcrustacea (Cladocera, Copepoda) with Macrophytes in Gala lake national park (in Turkish with English abstract). Trakya Univ. J.Sci. 8 (2), 109-114

Güher, H., Kırgız, T., 2008. Cladocera and Copepoda (Crustacea) Fauna of Gala Lake (Edirne) (in Turkish). KTU. 19 Ulusal Biyoloji Kongresi, 23-27 Haziran 2008, Trabzon, p.484

Güher, H., Kırgız,T.,Çamur, B., Güner, U., 2004. A Study on Zooplankton organisms community structures of Lake Terkos (Istanbul-Turkey). Pakistan Journal of Biological Sciences, 7 (4), 566-570.

Gündüz, E., 1997. A Checklist of Cladoceran species (Crustacea) living in Turkish inland waters (in Turkish with English abstract). Tr.J. Zoology 21.37-45.

Kiefer, F., 1952. Freilebende Ruderfusskrebse (Crustacea, Copepoda) aus Türkischen Binnengewasser. I. Calanoida I.U.F.F.Hidrobiyoloji Araştırma Enst. Yayınları. Seri B,I(2): 103-132

Kiefer, F., 1955. Freilebende Ruderfusskrebse (Crustacea, Copepoda) aus Türkischen Binnengewassern. II. Cyclopoida und Harpacticoida. I.U.F.F. Hidrobiyoloji Araş. Enst. Yayınları. Seri B, II(4): 108-132.

Lindeberg, K., 1953. Cyclopoides (Crustacés, Copépods) de la Turquie en particulier comme habitatns degrottes. I.U.F.F. Hidrobiyoloji Araştırma Enst. Yayınları Seri B,1(3): 149-185.

Mann, K.A., 1940. Über pelagische copepoden Türkischer Seen. Int. Rev. Ges. Hydrobiol. 40, 87 p.

Muckle, R., 1951. Cladoceran aus Turkischen binnengewasseren I. Ist. Univ. Fen Fak. Mec. 16. 367-387.

Okgerman, H.,Dorak, Z., Gürevin, C, Aktan, Y., 2007. Zooplankton distribution of Büyükçekmece lake and affecting environmental factors (in Turkish). XIV. Ulusal Su Ürünleri Sempozyumu, 04-07 Eylül 2007, Muğla, p.290.

Ortak, R., Kırgız, K., 1988. Cladocera and Copepoda (Crustacea) species of Gala Lake (in Turkish). IX. Ulusal Biyoloji Kongresi, 21-23 Eylül 1988, Sivas, Cilt 2: 377-385

Özçalpar, S., Temel, M., 2011. Seasonal changes in zooplankton community structure in Lake Küçükçekmece, Istanbul, Turkey. Turk J Zool. 35(5) 689-700 doi: 10.3906/zoo-1001-16

Segers, H., 2007. Annotated checklist of the rotifers (Phylum Rotifera), with notes on nomenclature, taxonomy and distribution. Zootaxa 1564, 104 pp.

Segers, H., 2008. Global diversity of rotifers (Rotifera) in freshwater. Hydrobiologia 595 49-59. doi: 10.1007/a10750-007-9003-7

Ustaoğlu, M.R., 2004. A Check-list for zooplankton of Turkish inland waters, E.U. Journal of Fisheries and Aquatic Sciences 21(3-4) 191-199.

Ustaoğlu, M.R., Altındağ, A., Kaya, M., Akbulut, N., Bozkurt, A., Özdemir Mis, D., Atasagun, S., Erdoğan, S., Bekleyen, A., Saler, S., Okgerman, H., 2012a. A checklist of Turkish Rotifers Turkish Journal of Zoology, 36 (1) 607-622. doi: 10.3906/zoo-1110-1

Ustaoğlu, M.R., Özdemir Mis, D., Aygen, C., 2012b. Observations on zooplankton in some lagoons in Turkey. J. Black Sea/Mediterranean Environment, 18, 2: 208-222.

Ustaoğlu, M.R., 2014. An updated zooplankton biodiversity of Turkish inland waters. FABA 2014: International Symposium on Fisheries and Aquatic Sciences, September 25-27, 2014, Trabzon, Turkey. p.386. 